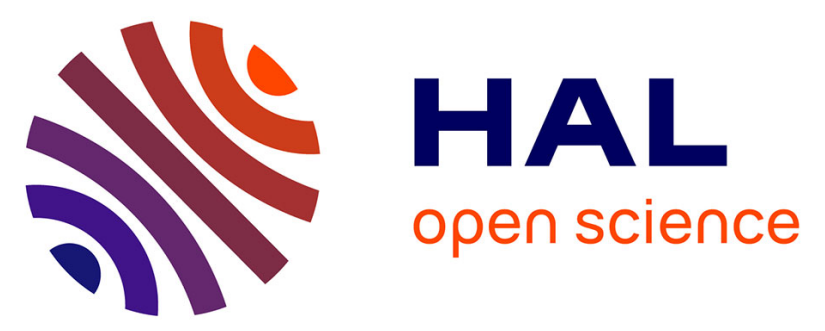

\title{
Entre maintien et transformation du caractère familial des exploitations agricoles. Le cas des exploitations laitières des Alpes du Nord
}

\author{
M. Terrier, S. Madelrieux, B. Dedieu
}

\section{- To cite this version:}

M. Terrier, S. Madelrieux, B. Dedieu. Entre maintien et transformation du caractère familial des exploitations agricoles. Le cas des exploitations laitières des Alpes du Nord. Gasselin, P.; Choisis, J.P.; Petit, S.; Purseigle, F.; Zasser, S. L'agriculture en famille: travailler, réinventer, transmettre. P. Gasselin, J.-P. Choisis, S. Petit, F. Purseigle et S. Zasser (éd.), EDP Sciences; INRA-SAD éd., pp.145-163, 2014, ISBN 978-2-7598-1192-2. 10.1051/978-2-7598-1192-2.c009 . hal-01118821

\author{
HAL Id: hal-01118821 \\ https://hal.science/hal-01118821
}

Submitted on 20 Feb 2015

HAL is a multi-disciplinary open access archive for the deposit and dissemination of scientific research documents, whether they are published or not. The documents may come from teaching and research institutions in France or abroad, or from public or private research centers.
L'archive ouverte pluridisciplinaire HAL, est destinée au dépôt et à la diffusion de documents scientifiques de niveau recherche, publiés ou non, émanant des établissements d'enseignement et de recherche français ou étrangers, des laboratoires publics ou privés. 


\title{
Entre maintien et transformation du caractère familial des exploitations agricoles. Le cas des exploitations laitières des Alpes du Nord
}

\author{
Médulline Terrier ${ }^{1}$, Sophie Madelrieux², Benoît Dedieu ${ }^{3}$
}

\section{Introduction}

L'agriculture familiale est aujourd'hui sous le feu des projecteurs. Tandis que les Nations unies ont proclamé 2014 "Année internationale de l'agriculture familiale », les plaidoyers visant à démontrer l'importance, en termes de développement, de cette forme d'exercice de l'activité agricole à travers le monde, trouvent à se faire entendre.

En France, l'exploitation familiale incarne un idéal longtemps mythifié par le dispositif idéologique syndical et consacré par les lois d'orientation de 1960 et 1962 (Bosse-Platière, 2005). L'expression " exploitation familiale " s'est cependant récemment vue effacée des textes de loi au profit de l'entreprise agricole (Rémy, 2010). Ce changement de vocable signe-t-il pour autant la disparition du lien entre la famille et l'activité agricole?

Les données statistiques récentes dressent à ce propos un tableau ambigu. Selon une définition largement partagée, l'exploitation familiale renvoie à une exploitation agricole sur laquelle la propriété et le contrôle de la gestion, transmis d'une génération à l'autre, sont dans les mains d'individus liés par la parenté ou par le mariage et qui réalisent le travail agricole (Gasson et al., 1993 ; Petit, 2006 ; Bjorkhaug et Blekesaune, 2008). Premier indice du maintien de la dimension familiale des exploitations françaises, c'est aujourd'hui $84 \%$ des actifs permanents qui sont familiaux ${ }^{4}$ (Agreste, 2012). Bien qu'en augmentation ces dernières années, l'appel à la main-

1. IRSTEA, DTM, BP 76, 38402 Saint-Martin d'Hères, France ;

INRA, UMR 1273 Métafort, 63122 St Genes Champanelle, France, terrier_medulline@yahoo.fr

2. IRSTEA, DTM, BP 76, 38402 Saint-Martin d'Hères, France, sophie.madelrieux@irstea.fr

3. INRA, UMR 1273 Métafort, 63122 St Genes Champanelle, France,

benoit.dedieu@clermont.inra.fr

4. Par actifs familiaux, sont désignés : les chefs d'exploitation, les coexploitants, les conjoints non coexploitants actifs ainsi que les autres membres de la famille actifs. Cette catégorie prend également en compte les salariés permanents familiaux.

Ce chapitre de l'ouvrage L'agriculture en famille : travailler, réinventer, transmettre est publié en Open Access sous licence creative commons CC-BY-NC-ND permettant l'utilisation non commerciale, la distribution, la reproduction 
d'œuvre extérieure à la famille demeure minoritaire. Par ailleurs, l'agriculture reste un métier à forte reproduction sociale puisque $87 \%$ des agriculteurs âgés de 21 à 34 ans ont un lien de parenté avec l'agriculteur qu'ils remplacent (Jacques-Jouvenot et Gillet, 2001) et dans les trois quarts des cas, il s'agit de leur père (Bessière et al., 2008). Si ce tableau statistique tend à confirmer le caractère familial de l'agriculture française contemporaine, certaines évolutions telles que l'augmentation des installations hors cadre familial et du travail extérieur des conjointes, la généralisation des formes sociétaires ou encore, le recours de plus en plus fréquent aux salariés (Olivier-Salvagnac et Legagneux, 2012) modèrent cet avis. En quoi ces évolutions transforment-elles le caractère familial des exploitations agricoles ? Comment coexistent ou s'articulent-elles avec le constat que nous faisons de permanence du caractère familial des exploitations françaises ?

Re-questionner aujourd'hui les rapports familiaux de production sur l'exploitation agricole peut néanmoins sembler trivial, voire éculé, tant l'exploitation familiale a été disséquée depuis 50 ans. Pourtant, à y regarder de plus près, la dimension familiale de l'exploitation agricole a peu à peu disparu du champ scientifique français. Avec les profondes transformations de leurs " objets " privilégiés - « le rural », le " paysan " et le "village " - les recherches des ruralistes français, très prolifiques des années 1960 aux années 1980, s’en sont peu à peu détournées (Alphandéry et Billaud, 2009). À la recherche de nouvelles grilles de lecture pour analyser les formes sociales de production et d'organisation de l'agriculture contemporaine, c'est aujourd'hui vers l'agriculture de firme que se tournent les sociologues agricoles (Purseigle, 2012). Ainsi, de façon paradoxale, l'" effacement " de la famille dans le traitement des questions agricoles pourrait laisser penser qu'exploitation et famille sont deux termes dont l'association ne fait aujourd'hui plus sens, et ce alors même que les statistiques nous invitent à penser le contraire.

Dans cette contribution, basée sur deux études réalisées dans les Alpes du Nord, nous voulons remettre les rapports familiaux de production au centre de l'analyse de façon à interroger les transformations du caractère familial des exploitations agricoles contemporaines. Si peu de travaux récents viennent amender les grilles de lecture produites par les ruralistes (voir par exemple : Bessière, 2010 et Dahache, 2012), nous faisons l'hypothèse que ces recherches, bien que socialement datées pour la plupart, proposent des catégories d'analyse pertinentes pour penser les rapports de production et leurs transformations sur l'exploitation familiale d'aujourd'hui.

Ce chapitre s'organise en trois parties. Dans une première partie, nous montrerons que l'exploitation familiale telle qu'elle fut pensée et décrite dans les années 1960 et 1970 constitue un idéal-type à partir duquel penser les transformations à l'œuvre. Dans une deuxième partie, après avoir présenté les grandes lignes des deux études de cas sur lesquelles se base notre propos, nous nous interrogerons sur ce qui distingue les exploitations enquêtées de l'idéal-type précédemment construit. Nous explorerons ainsi quelques-unes des évolutions récentes qui tendent à « défamilialiser " l'exploitation agricole et nous conclurons, dans une troisième partie, que c'est aujourd'hui en termes de diversité et de complexité qu'il faut penser la dimension familiale de l'exploitation agricole. 


\section{L'exploitation agricole familiale des années 1970 comme idéal-type pour penser les transformations contemporaines des rapports familiaux de production}

Depuis la Seconde Guerre mondiale, l'agriculture en France est politiquement et juridiquement définie comme une activité familiale. La loi d'orientation agricole (LOA) de 1960 soutient à la fois la "modernisation " et l'insertion de l'agriculture dans l'économie de marché, mais elle promeut aussi une structure familiale de production. Le modèle de "l'exploitation à 2 UTH (unités de travail humain)" telle que définie par les LOA de 1960 et 1962 devait permettre à un ménage de subsister et d'atteindre une parité économique et sociale avec le reste de la société, sans recours au travail extérieur (Rémy, 1987). Vingt ans plus tard, la LOA de 1980 réaffirme la dimension familiale de l'activité agricole en stipulant que " les exploitations familiales à responsabilité personnelle [...] constituent la base de l'agriculture française " (Bosse-Platière, 2005).

Si les politiques de modernisation et de professionnalisation de l'agriculture menées par l'État depuis les années 1960 se sont attachées à " défamilialiser " les rapports de production au sein de l'exploitation agricole (création des groupements agricoles d'exploitation en commun [GAEC] puis des exploitations agricoles à responsabilité limitée [EARL], politique d'aides à l'installation, etc.), il n'en demeure pas moins que l'agriculture constitue un domaine d'activité particulier en ce sens qu'il s'y joue tout à la fois des rapports professionnels et des rapports familiaux (Barthez, 1982).

Des années 1960 aux années 1980, les sociologues ruralistes se sont attachés à rendre compte de cette imbrication des logiques professionnelle et familiale sur l'exploitation agricole en s'appuyant sur des grilles de lecture de tradition structuraliste. Leurs recherches décrivent une famille agricole traversée de rapports de domination, et dont le mode de fonctionnement spécifique se caractérise par une faible autonomie des individus : leurs comportements sont déterminés par leur statut dans la famille, tandis qu'une division du travail hiérarchisée définit des rôles différenciés et complémentaires notamment entre hommes et femmes et entre générations (Van de Walle, 1993).

À l'heure où la sociologie du travail et de la famille constate l'émergence d'une famille conjugale relationnelle et individualiste ainsi que l'importance grandissante de la norme de l'émancipation personnelle au travail, par opposition avec l'assignation identitaire à un destin professionnel tout tracé (De Singly, 2005 ; Bessière, 2006 ; Giraud, 2006), les analyses produites par les ruralistes pourraient apparaitre passéistes. Cependant, et c'est là notre hypothèse de travail, pour datée qu'elle soit, cette littérature pose les bases conceptuelles d'une analyse du fonctionnement familial des exploitations et suggère des éléments dont la variabilité dans le temps et entre les exploitations permet de saisir la nature et la diversité du caractère familial des exploitations contemporaines. En décrivant ainsi les exploitations agricoles dans un " excès de famille ", leurs auteurs dessinent une exploitation familiale idéaltypique, au sens wébérien de catégorie organisatrice de la réalité pour aider à penser, 
et à partir de laquelle il devient possible de repérer et de caractériser les transformations à l'œuvre aujourd'hui. C'est l'objet des paragraphes suivants que de dégager les caractéristiques de cet idéal-type.

Cette exploitation familiale que nous érigeons en idéal-type renvoie donc à l'exploitation des années 1960-1970 : plus vraiment paysanne car sur le chemin de la modernisation et de la " professionnalisation ", mais encore définitivement familiale (Mundler et Rémy, 2012).

Sur cette exploitation familiale idéal-typique, les rapports sociaux sont décrits comme relevant d'un choc entre des rapports familiaux et des rapports professionnels (Barthez, 1982). Au-delà de l'imbrication des sphères professionnelle et familiale, ce sont également différentes temporalités qui régissent les interactions des individus au sein des familles. En effet, dans ces familles agricoles, non seulement la socialisation professionnelle et la socialisation familiale sont indissociables, mais encore, la famille y joue en même temps un rôle de reproduction (reproduction professionnelle et transmission patrimoniale) et un rôle de coproduction (production professionnelle et production domestique) (Gramain et Weber, 2003). On a là deux points d'entrée qui rendent compte du caractère familial de l'exploitation agricole : (i) la transmission et (ii) la perméabilité des sphères domestique et professionnelle.

\section{La transmission : un processus multidimensionnel, clé de voûte de la dimension familiale de l'exploitation}

Nous l'avons évoqué en introduction, le groupe socioprofessionnel des agriculteurs est l'un de ceux dont la reproduction s'appuie le plus sur l'héritage (DubuissonQuellier et Giraud, 2010). Ce constat - l'agriculture présente la particularité de recruter ses membres dans la famille - en appelle un autre, celui de l'importance de la transmission du métier, au sein des familles agricoles, mais aussi des conditions nécessaires à l'exercice de ce métier, c'est-à-dire des moyens de production. La transmission familiale, processus structurant du fonctionnement et de la continuité des exploitations agricoles, renvoie, en même temps, à la passation, de génération en génération de trois éléments (Bessière, 2003) : (i) un patrimoine physique et symbolique, à la fois moyens de production et histoire d'une famille, (ii) un métier qui met en jeu des savoir-faire spécifiques, et (iii) un statut de chef d'exploitation, c'est-à-dire d'une autorité sur la ferme.

En effet, pour devenir agriculteur, il ne suffit pas, comme dans la plupart des autres métiers, d'acquérir une qualification professionnelle, il faut avant tout pouvoir disposer d'un droit d'usage sur l'ensemble des moyens de production : du foncier agricole, des équipements, des bâtiments, un cheptel, des capitaux financiers, etc. (Barthez, 1982). Une première dimension de la transmission en agriculture renvoie donc à l'héritage en tant que bien physique, à la fois outil de travail et patrimoine familial. De par sa dimension patrimoniale, l'héritage transmis n'est pas seulement physique, il renvoie à tout un ensemble d'éléments matériels et immatériels qui 
concourent à maintenir l'identité et l'autonomie de l'agriculteur dans le temps et dans l'espace (Ollagnon, 1989). Les " moyens de production-patrimoine " lient ainsi les générations les unes aux autres parce qu'ils contiennent l'histoire et la mémoire familiale, ainsi que le travail investi par plusieurs générations d'éleveurs (Jacques-Jouvenot, 1997).

Outre l'héritage des biens physiques, la transmission patrimoniale met également en jeu la passation du métier d'agriculteur. Cette dernière renvoie à l'acquisition, par au moins l'un des enfants de la fratrie, d'un goût pour l'agriculture et de savoir-faire spécifiques, bien en amont de la reprise effective : " en plus de l'héritage transmis, les héritiers bénéficient du fait d'avoir été profilés, socialisés pour être héritiers " (Bertaux, 1977). À la transmission d'un patrimoine professionnel et familial se joint celle de savoirs, d'une organisation du monde, de l'histoire d'un métier qui s'inscrit dans un processus de socialisation agricole (Salmona, 1994), et qui passe par le travail en commun, à la fois sur la ferme et en famille, et ce dès l'enfance (JacquesJouvenot, 1997). Mais, la logique familiale de reproduction du métier, se heurte à la notion d'installation agricole qui reconnait l'agriculteur apte à produire selon des normes établies par l'État, hors du rapport familial.

À ces deux premières dimensions de la transmission s'en ajoute une troisième, celle du statut de chef d'exploitation, c'est-à-dire des pouvoirs et de l'autorité donnant légitimité professionnelle à celui qui succède. Le statut de professionnel du fils ne devient en effet réel qu'à partir du moment où le père accepte de partager son autorité sur l'exploitation (comme ce peut être le cas dans les associations) ou bien de transmettre l'intégralité de son autorité (installation du fils, départ du père en retraite). S’il existe une diversité de modalités pratiques quant au processus de passation des moyens de production, "la transmission est un processus réciproque où les choix du fils se heurtent et influent nécessairement sur les stratégies du père. [...] Négociations, stratégies, soumission, révolte [sont] autant d'interactions familiales constitutives des formes et modalités de la transmission des exploitations en agriculture " (Jacques-Jouvenot, 1997). Il n'est pas rare que, sur notre exploitation familiale idéal-typique, le fils futur repreneur doive travailler en tant quaide familial pendant plusieurs années en attendant que son père parte à la retraite, ou encore, que ce premier reste sous le joug de l'autorité paternelle bien après que le père soit retraité.

\section{Au quotidien : la perméabilité des sphères familiale et professionnelle}

Une autre des caractéristiques les plus prégnantes de l'agriculture est la grande porosité, au quotidien, entre les sphères familiale et professionnelle, du fait de la participation des membres de la famille à l'élaboration de la production, mais aussi de la proximité des lieux permettant également une grande perméabilité des temps.

En effet, sur l'exploitation familiale idéal-typique, le travail est effectué par un groupe d'individus qui ne sont pas réunis selon une logique d'embauche et de sélection sur la base de compétences professionnelles sur le marché du travail, mais au 
contraire, selon une logique de développement de la famille. L'organisation du travail agricole, la répartition des tâches et des responsabilités au sein du collectif de travail, implique une répartition de l'autorité, une division des tâches selon des critères empruntés à la vie familiale (critères biologiques tels que âge, sexe et statut de chacun dans la parenté) et non à la profession (Barthez, 2005).

Cependant, cela ne signifie pas pour autant que tous les membres du collectif de travail soient reconnus par un statut professionnel. Au contraire, le travail agricole se déployant à partir d'une structure familiale, l'activité agricole emprunte des caractères de l'activité domestique comme le témoigne la notion "d'aide familiale ». Le collectif de travail agricole se compose ainsi d'un chef d'exploitation et d'aides familiaux. Les travailleurs qui entourent le chef d'exploitation, son épouse et ses enfants, ainsi nommés à partir de la référence familiale, contribuent, sans statut professionnel, à l'élaboration de la production agricole. Par ailleurs, le statut de chef d'exploitation est sexué. Le travail de la femme dans l'exploitation agricole, souvent peu visible, apparait comme une extension des activités domestiques et non comme une activité professionnelle. Les épouses qui ne travaillent pas à l'extérieur sont sans professions ou plutôt cent professions (Bessière, 2008) : elles apportent au collectif familial du travail non professionnellement reconnu (Delphy, 1983).

Dans cette exploitation familiale, on observe également un recouvrement spatial et temporel des scènes domestiques et productives. La maison d'habitation, parfois plurigénérationnelle, et le siège d'exploitation sont bien souvent au même endroit, ce qui concoure à la non séparation des vies familiale et professionnelle, caractéristique du " labeur paysan » (Barthez, 1986).

\section{Lecture des rapports sociaux sur l'exploitation familiale : les dimensions en jeu}

De cette description de l'exploitation agricole dont témoignait la sociologie rurale il y a une vingtaine d'années, émergent différentes dimensions (nous en retenons sept) qui permettent de caractériser ce à quoi renvoie le caractère familial (Fig. 1). Trois de ces dimensions ont trait à la transmission patrimoniale (i) des moyens de production, (ii) du métier et (iii) du statut de chef d'exploitation ; les quatre autres renvoient à (iv) la co-production agricole familiale sur la ferme, (v) à la reconnaissance professionnelle de l'épouse, et à la porosité (vi) des temps et (vii) des lieux de vie et de travail.

Dans la partie suivante, nous allons, à partir de deux études de cas conduites dans les Alpes du Nord, nous interroger sur le positionnement des exploitations enquêtées par rapport à l'idéal-type construit : dans quelle mesure, et comment, les exploitations étudiées s'en éloignent-elles? Quelles sont les nouvelles modalités d'articulation entre la famille et l'exploitation ? Comment coexistent, sur ces exploitations, permanence du caractère familial et " défamilialisation " ? 


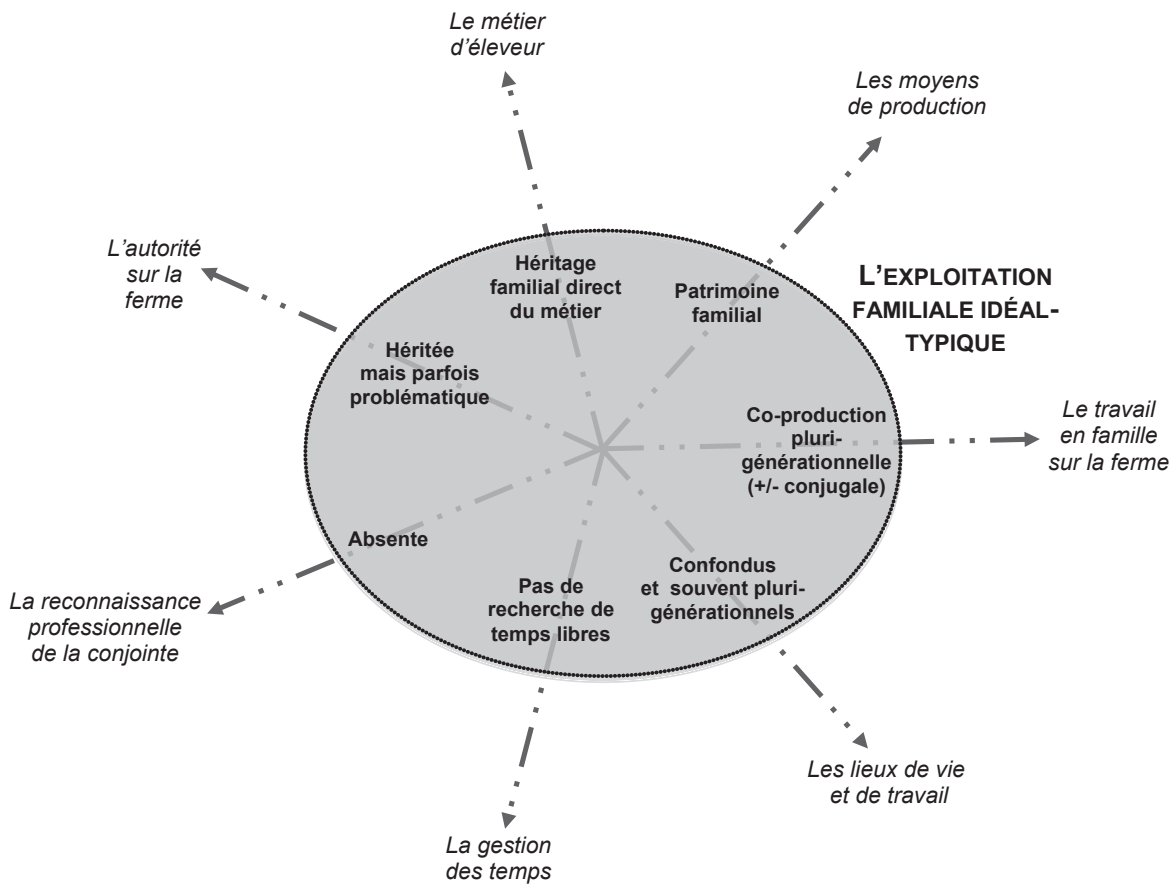

Fig. 1. Représentation de l'idéal-type de l'exploitation familiale à partir des dimensions identifiées pour rendre compte des rapports familiaux de production sur l'exploitation agricole.

\section{Le caractère familial des exploitations à l'épreuve. Étude de cas dans les Alpes du Nord}

\section{Lecture transversale des évolutions du caractère familial des exploitations agricoles à partir de deux études de cas}

L'exposé qui suit propose une lecture transversale de deux études de cas conduites dans les Alpes du Nord à partir d'une analyse approfondie d'un nombre restreint de situations contrastées. Bien que menées dans des contextes de recherche différents, ces deux études se sont attachées à explorer les transformations du caractère familial des exploitations.

La première étude fut menée dans le cadre d'un travail doctoral ${ }^{5}$ qui visait à rendre compte de la coévolution entre les configurations familiales et les trajectoires des

5. Le travail a bénéficié du soutien du projet MOUVE ANR-10-STRA-005- 01. 
exploitations d'élevage sur le plateau du Vercors (Isère et Drôme) (Terrier et al., 2012 ; Terrier, 2013). La deuxième étude ${ }^{6}$, conduite dans les Bauges (Savoie), s'est intéressée à la pénétration du salariat non familial dans les exploitations en se basant sur une analyse du recours au salariat et de son inscription dans l'évolution des relations entre la famille et l'exploitation (Madelrieux et al., 2009).

Plusieurs traits communs à ces deux recherches autorisent une lecture transversale. Elles s'ancrent en effet dans un même contexte territorial et productif dominé par les exploitations laitières de moyenne montagne préalpine. Soulignons que les exploitations d'élevage bovin lait en général font fortement appel à la main-d'œuvre familiale, notamment en raison du travail d'astreinte auprès des animaux (Dedieu et al., 1998). Le travail en couple y est également plus fréquent que dans d'autres secteurs de production (Giraud et Rémy, 2008).

Par ailleurs, la méthodologie adoptée repose, dans les deux cas, sur des entretiens semi-directifs, de type "récits de vie ", auprès d'une diversité d'acteurs de l'exploitation familiale en vue d'une analyse croisée des enjeux et expressions de l'articulation entre famille et exploitation. Dans le Vercors, nous avons ainsi rencontré en plus des éleveurs, leur conjoints et, lorsque cela était possible, leurs ascendants (15 personnes rencontrées sur 7 exploitations) ; dans les Bauges, ce sont les exploitants et les salariés qui ont été interviewés (13 exploitants et 9 salariés rencontrés sur 13 exploitations). Les données recueillies devaient permettre de saisir quelle était la place de chacun par rapport à l'entreprise agricole et de recueillir la diversité des points de vue et des représentations au sein même des familles et des collectifs de travail. Faisant l'hypothèse que les rapports familiaux de production s'expriment également à travers les choix techniques sur l'exploitation agricole, nous avons par ailleurs retracé les trajectoires des exploitations d'élevage en utilisant des outils méthodologiques issus des sciences agronomiques.

Dans les deux cas, des situations contrastées ont été choisies afin de témoigner de la pluralité des rapports qu'entretiennent aujourd'hui les familles agricoles avec l'entreprise. Pour ce faire, outre des exploitations engageant les deux membres du couple sur l'exploitation agricole, des associations entre pairs familiaux mais aussi non familiaux, des situations de pluriactivité familiale, de salariat ou encore de gestion pluri-générationnelle furent étudiées, le point commun entre toutes étant la conduite d'un atelier d'élevage bovin lait, avec ou non transformation du lait à la ferme.

\section{Évolutions du caractère familial des exploitations agricoles dans les Alpes du Nord}

Les sept dimensions retenues dans la première partie de ce chapitre pour décrire le caractère familial des exploitations et leurs évolutions se déclinent, sur nos terrains, selon une diversité de réalités. Après avoir identifié l'ensemble des modalités

6. Le travail a bénéficié du soutien du projet TRANS ANR-05-PADD-003. 
que peuvent prendre ces dimensions, nous appliquerons la grille de lecture ainsi construite à quelques-unes des exploitations étudiées de façon à mettre en évidence comment s'y articulent les deux tendances que sont le maintien du caractère familial et la " défamilialisation".

\section{L'inscription de l'exploitation agricole dans une lignée patrimoniale : une réalité polymorphe}

Si, dans l'échantillon étudié, la grande majorité des éleveurs exercent, sur la ferme familiale, le même métier que leur père, on observe néanmoins que tous n'entretiennent pas le même rapport à leur patrimoine.

Sur l'exploitation familiale idéal-typique, la transmission, d'une génération à l'autre, des moyens de production, du métier et du statut de chef d'exploitation est constitutive des rapports sociaux sur la ferme. La description que nous en faisions plus haut renvoie au vécu de plusieurs des éleveurs rencontrés. C'est par exemple les cas de Jean Martin ${ }^{7}$ et de Jacques Bernard, deux éleveurs d'une quarantaine d'années qui se sont installés sur la ferme familiale à la suite de leur père après avoir travaillé plusieurs années en tant quaide familial avant de pouvoir accéder au statut de chef d'exploitation. On remarque également, lorsque l'on s'intéresse à l'évolution des moyens de production et des pratiques d'élevage, que très peu de choses ont été modifiées sur la ferme depuis leur installation. L'exploitation d'aujourd'hui, son capital, et la façon dont y est géré l'élevage, sont ainsi fortement marqués par les générations précédentes qui, par leurs choix d'investissement, ont transmis un outil de production qui, par la suite, n'a pu être que très peu modifié. Et cet investissement de la génération précédente dans la reprise de l'exploitation familiale s'exprime encore aujourd'hui dans le discours des protagonistes sous la forme d'un " devoir moral ». Lépouse de Jean Martin témoigne par exemple de l'importance du maintien de la ferme familiale pour son époux, et ce, malgré les difficultés économiques.

"S'il fallait qu'il arrête et faire autre chose ... à un moment j'ai cru, parce qu'il parlait justement d'arrêter les vaches quand le lait marchait pas. [...]Mais ...voilà, après c'est remonté, c'est resté quoi. [...] Je pense que le fait que c'est à ses grands-parents, à ses parents. Après à lui, je pense ça y fait oui. Laisser tomber le truc que ses grands-parents ont mis en place... voilà je pense qu'il penserait que ses parents seraient déçus qu'il lâche tout." "

Cette prégnance, réelle et/ou symbolique, de la génération précédente sur l'exploitation actuelle peut également se traduire par un fort rapport d'autorité entre les générations. Plusieurs éleveurs entretiennent en effet de difficiles relations avec leur père, qui bien que retraité depuis des années, reste très investi sur la ferme et dans les décisions. On a dans ces cas, une tension entre la transmission légale de l'exploitation qui instaure l'éleveur autonome vis-à-vis de ses décisions à gérer l'exploitation, et la transmission pratique, retrait des parents.

7. Les noms des personnes interviewées ainsi que les noms de lieu ont été modifiés afin de préserver l'anonymat des enquêtés. 
Pour d'autres éleveurs au contraire, si l'exploitation héritée constitue une assise fondamentale à leur activité actuelle, elle se dilue progressivement au cours de la trajectoire, au gré des investissements réalisés. Ces cas témoignent d'une toute autre configuration des trois dimensions de la transmission dans laquelle les biens hérités prennent une importance relative et le rapport d'autorité entre les générations est assoupli. L'exemple du GAEC Dumont, sur lequel le père préfere se retirer de l'association et recréer une structure individuelle de façon à laisser à ses deux fils la totale autorité, illustre bien ce cas. Ces derniers ont en effet une vision très différente de leur père quant à la façon de faire de l'élevage :

Enquêtrice (E) : Et du coup, votre frère, il a pris la place de votre père, ou... c'est... Julien Dumont (JD) : Oui, oui, oui. Oui, parce qu'avec mon père, il y a plus... conflit de génération de...

$\mathrm{E}:$ Entre vous deux? Enfin... entre votre père et vous?

JD : Oui, oui. On en faisait bien assez... enfin, je veux dire, je pense qu'on ne pense pas pareil à cinquante ans qu'à... qu'à 25, 30 ans et... euh... on veut aller de l'avant, on veut aller de l'avant, en faire toujours plus, et là... Donc...

$\mathrm{E}$ : Votre père ne voulait pas trop s'agrandir, quoi?

JD : Oui, voilà, c'est... c'est moi qui ai poussé en gros, hein, on va dire.

Certains des éleveurs rencontrés n'ont pas succédé à leurs parents sur la ferme familiale. Ne pas reprendre une ferme familiale n'efface toutefois pas forcément la référence à la transmission. Parmi ces éleveurs, si certains d'entre eux ne sont pas issus de familles agricoles et ont acquis, et non hérités, les moyens de production dont ils disposent, ce n'est pas le cas de Gabriel Collin et de Nicolas Ollivier. Tous deux, bien qu'éleveurs sur une structure qu'ils ont monté de toutes pièces, ont en effet bénéficié d'une socialisation agricole en tant que fils cadet (dans le cas de Gabriel Collin) et de neveu et petit fils (Nicolas Ollivier) d'éleveur. La trajectoire du GAEC qu'ils ont constitué ensemble donne de plus à voir l'importance qu'a eue, au début de la trajectoire, l'exploitation des parents de Gabriel Collin reprise par son frère aîné. C'est en effet grâce à l'assise matérielle (bâtiments et équipements) de l'exploitation familiale que l'éleveur a pu créer sa propre exploitation.

L'analyse des situations de salariat met également en exergue la diversité des façons dont s'articulent les trois dimensions de la transmission. Certains des salariés rencontrés, tout en s'inscrivant dans un processus de reprise de la ferme familiale, choisissent de se salarier un temps sur d'autres exploitations dans l'attente du départ à la retraite de leurs parents (pour ainsi récupérer en même temps les moyens de production et l'autorité sur la ferme), mais également pour diversifier leurs expériences et leurs savoirs. Cette phase transitoire de salariat est aussi vécue comme un temps d'épargne en vue de l'acquisition des moyens de production familiaux, de mise à distance de sa propre famille et d'insertion dans des réseaux de connaissance professionnels. Autant d'éléments qui témoignent d'une professionnalisation distanciée par rapport à la famille, via la confrontation à d'autres réalités que ce qu'ils connaissent de l'exploitation familiale et qui favorisent une réflexivité sur leur propre projet, comme en témoigne Damien Dupont. 
"Bien sûr moi je veux pas minstaller comme... jaurais pu minstaller [...] Mais j’ai pas envie de faire ça pressé, il faut que ce soit réfléchi, il faut pas sinstaller n’importe comment. [...] Donc comme ça, ça me fait plein de... je vois plein de choses aussi ».

À l'inverse, des exploitants n'ayant pas de repreneur familial peuvent se tourner vers l'embauche d'un salarié pour espérer, à terme, son installation et le maintien de la structure. Il peut alors se mettre en place un processus "d'adoption " qui relève des références à la famille (Barthez, 1999).

De cet exposé, il émerge ainsi que les trois dimensions de la transmission patrimoniale, que nous avions identifiées comme constitutive du caractère familial de l'exploitation idéal-typique, se déclinent et s'articulent différemment selon les cas. Pour chacune, trois modalités permettent de caractériser un gradient dans le rapport des éleveurs au patrimoine familial (Tab. 1).

\section{Tab. 1. Modalités décrivant le caractère familial des exploitations du point de vue de la transmission.}

\begin{tabular}{ll}
\multicolumn{1}{c}{ Modalités } & $\begin{array}{l}\text { 1. L'exploitation } \\
\text { familiale idéal- } \\
\text { typique : coincidence } \\
\text { de la ferme et de la } \\
\text { famille }\end{array}$
\end{tabular}
Les moyens de
production

Le métier

L'autorité sur la ferme

Héritage direct de père en fils

Héritée (l'éleveur est chef d'exploitation sur l'exploitation familiale) mais les relations sont difficiles entre les générations

\section{Cas intermédiaires}

Issus d'un patrimoine familial transmis mais transformés par des investissements ultérieurs

Héritage indirect du métier : l'éleveur a bénéficié d'une socialisation agricole dans la famille élargie (oncle, grands-parents, etc.)

3. L'exploitation et la famille : deux mondes séparés

Héritée, mais le rapport d'autorité entre les générations est assoupli. Il peut même y avoir un retrait des parents de façon à laisser aux enfants installés la totale autorité sur la Pas d'endoreproduction : l'éleveur n'est pas issu d'une famille agricole

Les moyens de production ne sont pas issus d'un héritage familial

Acquise en dehors de toute référence familiale (concerne également les fils d'éleveur installés sur une autre ferme que la ferme familiale) ferme 


\section{Une diversité de configurations familiales au quotidien}

Cette première lecture, du point de vue de la transmission, du caractère familial des exploitations doit néanmoins être complétée par celle des interactions au quotidien entre famille, collectif de travail et exploitation. Le collectif de travail sur l'exploitation met bien souvent en jeu la génération précédente, mais également les conjoints, et parfois des salariés, dont nous avons cherché à comprendre les implications objectives et les vécus subjectifs.

En analysant les cas étudiés à partir de l'examen des quatre dimensions retenues, nous mettons au jour des situations contrastées, témoignant de fonctionnements familiaux différents et d'une distanciation plus ou moins grande avec l'exploitation. On observe ainsi une variété de situations de travail des conjointes d'exploitants.

Certaines femmes travaillent sur l'exploitation, avec ou sans statut agricole reconnaissant leur contribution au travail productif, et selon des degrés d'investissement très variés, allant du simple coup de main à la prise en charge intégrale d'un atelier de production.

La mise en regard des cas de Nicole Martin, Laurence Dubois et Annie Durand est ici illustrative. Nicole Martin, bien que travaillant sur l'exploitation de son mari (aide régulière au travail d'astreinte et de saison) et n'exerçant pas d'autre activité professionnelle, ne bénéficie pas d'un statut agricole. L'aide apportée au travail agricole constitue un travail d'exécution ; le pouvoir d'orchestration (SalifiosRothschilf, 1976), concernant les décisions importantes qui orientent la conduite de l'exploitation, restant détenu par son mari. La situation s'apparente alors à celle d' " aide familiale " (telle que nous la décrivions dans la première partie) : non reconnue professionnellement, elle contribue à titre d'épouse à l'activité de son mari. Au contraire, Laurence Dubois bénéficie du statut de conjoint collaborateur alors même qu'elle exerce un trois-quarts temps à l'extérieur et n'aide que rarement sur la ferme. Enfin, Annie Durand travaille à plein temps en couple sur l'exploitation. Au bout de vingt ans, elle a échangé son statut de conjoint collaborateur avec celui de chef d'exploitation de son mari, de façon à ce que tous deux bénéficient des droits sociaux associés au statut d'agriculteur.

Enfin, pour quelques-unes d'entre elles, l'exploitation familiale ne représente rien de plus que le lieu de travail de leur conjoint, ces dernières exerçant leur propre activité professionnelle et ne participant jamais au travail agricole. Cette distance de la famille à l'exploitation dont témoigne l'absence de coproduction conjugale sur la ferme, ainsi que l'autonomie professionnelle dont disposent les conjoints dans cette dernière situation, est renforcée par la distance géographique entre les lieux de vie et de travail et le cloisonnement temporel instauré entre les scènes. La répartition et la réalisation du travail sur ces fermes sont réfléchies et organisées de façon à libérer du temps libre pour soi ou pour la famille.

Cette frontière entre vies professionnelle et familiale est toutefois loin d'être la norme dans notre échantillon et la coïncidence de la ferme et de la maison telle qu'elle caractérise l'idéal-type de l'exploitation familiale, encore fréquente, génère une diversité de pratiques quant à la gestion des temps et de l'espace. Nous pouvons 
ici poursuivre la comparaison, que nous amorcions plus haut, des cas des exploitations individuelles des familles Dubois et Martin, qui toutes deux se confondent géographiquement avec la maison d'habitation. La façon dont sont gérés les temps libres, témoigne en effet de la centralité de l'exploitation pour la famille Martin. Nicole évoque par exemple très longuement et à plusieurs reprises l'impossibilité de partir en vacances en famille et le poids que cela représente pour elle :

"Il y a tout le temps quelque chose! Mais pour tout. Les enterrements de ses parents, il s'est jamais fait remplacer. On s'est mariés, il s'est pas fait remplacer. On a marié notre fille en septembre l'année dernière et bon au lieu d'aller à l'apéro avec nous, ben il est venu traire. [...] Il y a beaucoup de choses qu'on fait pas à cause de ça. On fait pas... Il faut avoir le moral. Franchement, il faut avoir le moral. Il y a des fois... "

Au contraire, le couple Dubois cherche à maintenir une certaine distance entre la ferme et la vie de famille qui passe par la prise régulière de week-ends, de vacances, mais aussi par leur refus de s'investir à deux sur l'exploitation. L'emploi salarié de Laurence est apprécié à double titre : d'une part, il apporte un revenu fixe, qui desserre l'obligation pour la ferme de dégager un revenu suffisant pour faire vivre la famille, et d'autre part, il permet un certain cloisonnement des mondes professionnels et, par là-même, une dissociation entre travail et non travail. Cette configuration des relations se traduit d'ailleurs concrètement par le choix qu'a fait le couple d'investir d'abord dans la construction d'une maison d'habitation séparée de celle des parents de l'éleveur, et seulement par la suite dans un nouveau bâtiment d'élevage.

Les entretiens réalisés rendent également compte de la façon dont le recours au salariat vient, au quotidien, renforcer cette coproduction familiale ou au contraire appuyer la distanciation entre la famille et l'exploitation. Parfois transitoire pour faire face à la diminution de l'implication de la conjointe à certaines périodes du cycle de la vie familiale (grossesse, période où les enfants sont en bas âge), il peut également témoigner d'une évolution des liens entre la famille et l'exploitation. C'est par exemple le cas lorsque l'épouse ne souhaite pas s'impliquer sur l'exploitation lors du départ des parents, main-d'œuvre dont l'éleveur ne peut faire l'économie. Le salariat peut également être perçu comme une solution à une charge de travail trop élevée qui permet de dégager davantage de temps pour la vie de famille, comme en témoigne Gérard Moreau :

"Je crois que l'amélioration de la vie de famille a un coût (celui du salarié), et la santé, parce que, bosser tous les jours de l'année, ce n'est pas possible non plus, au bout d'un moment ça craque ".

Toutefois la charge financière, ainsi que le contrat de travail liant employeur et salarié, ne permettent pas une substitution totale de la main-d'œuvre familiale non statutairement reconnue et qui ne compte pas ses heures. De ce fait, le salariat est davantage pensé par les éleveurs rencontrés comme une étape vers l'association voire, lorsqu'il n'y a pas de repreneur dans la famille, vers la transmission. Mais la question de la bonne distance à tenir entre patron et salarié demeure souvent problématique du fait de la référence souvent bien présente au fonctionnement familial et aux 
pratiques qui lui sont liées (l'exigence d'être flexible et/ou de ne pas trop compter ses heures, la prise des repas avec la famille, l'implication dans les décisions, etc.).

Pour rendre compte de cette diversité de fonctionnement des familles agricoles au quotidien, nous retenons les modalités présentées dans le tableau 2.

Tab. 2. Modalités décrivant le caractère familial des exploitations du point de vue de la perméabilité des sphères familiale et professionnelle.

\begin{tabular}{|c|c|c|c|}
\hline Modalités & $\begin{array}{l}\text { 1. L'exploitation } \\
\text { familiale idéal- } \\
\text { typique : coüncidence } \\
\text { de la ferme et de la } \\
\text { famille }\end{array}$ & $\begin{array}{l}\text { 2. Cas } \\
\text { intermédiaires }\end{array}$ & $\begin{array}{l}\text { 3. L'exploitation } \\
\text { et la famille : deux } \\
\text { mondes séparés }\end{array}$ \\
\hline $\begin{array}{l}\text { Les lieux de vie et de } \\
\text { travail }\end{array}$ & $\begin{array}{l}\text { Confondus et pluri- } \\
\text { générationnels }\end{array}$ & $\begin{array}{l}\text { Confondus mais une } \\
\text { séparation claire est } \\
\text { instaurée entre les } \\
\text { lieux de résidence } \\
\text { des différentes } \\
\text { générations }\end{array}$ & $\begin{array}{l}\text { Séparés } \\
\text { géographiquement }\end{array}$ \\
\hline $\begin{array}{l}\text { Le travail en famille } \\
\text { sur la ferme }\end{array}$ & $\begin{array}{l}\text { Co-production pluri- } \\
\text { générationnelle (et } \\
\text { souvent conjugale) }\end{array}$ & $\begin{array}{l}\text { Co-production } \\
\text { conjugale }\end{array}$ & $\begin{array}{l}\text { Le collectif de travail } \\
\text { ne met en jeu aucun } \\
\text { membre de famille } \\
\text { (autre que le ou les } \\
\text { éleveur(s)) }\end{array}$ \\
\hline $\begin{array}{l}\text { La reconnaissance } \\
\text { professionnelle de la } \\
\text { conjointe }\end{array}$ & Absente & $\begin{array}{l}\text { La contribution au } \\
\text { travail agricole de } \\
\text { l'épouse est reconnue } \\
\text { statutairement }\end{array}$ & $\begin{array}{l}\text { Autonomie } \\
\text { professionnelle : } \\
\text { si l'épouse } \\
\text { travaille parfois } \\
\text { ponctuellement } \\
\text { sur la ferme, elle } \\
\text { exerce surtout sa } \\
\text { propre activité } \\
\text { professionnelle à } \\
\text { l'extérieur }\end{array}$ \\
\hline La gestion des temps & $\begin{array}{l}\text { Inexistante : pas de } \\
\text { distinction entre les } \\
\text { temps de travail et les } \\
\text { temps libres }\end{array}$ & $\begin{array}{l}\text { Existante mais non } \\
\text { structurante du } \\
\text { fonctionnement de } \\
\text { l'exploitation }\end{array}$ & $\begin{array}{l}\text { Recherchée et } \\
\text { organisée }\end{array}$ \\
\hline
\end{tabular}




\section{Une grille de lecture qui rend compte de l'évolution du caractère familial dans l'histoire de l'exploitation}

Appliquée aux cas étudiés, cette grille de lecture permet de rendre compte, à l'échelle des exploitations, de la façon dont a évolué le caractère familial. Prenons par exemple les cas déjà évoqués des familles Martin et Dubois. La représentation graphique de la grille de lecture aux situations initiales (à l'installation de l'éleveur) et actuelles des exploitations (Fig. 2) montre, dans chacun des deux cas, l'évolution des rapports familiaux de production.
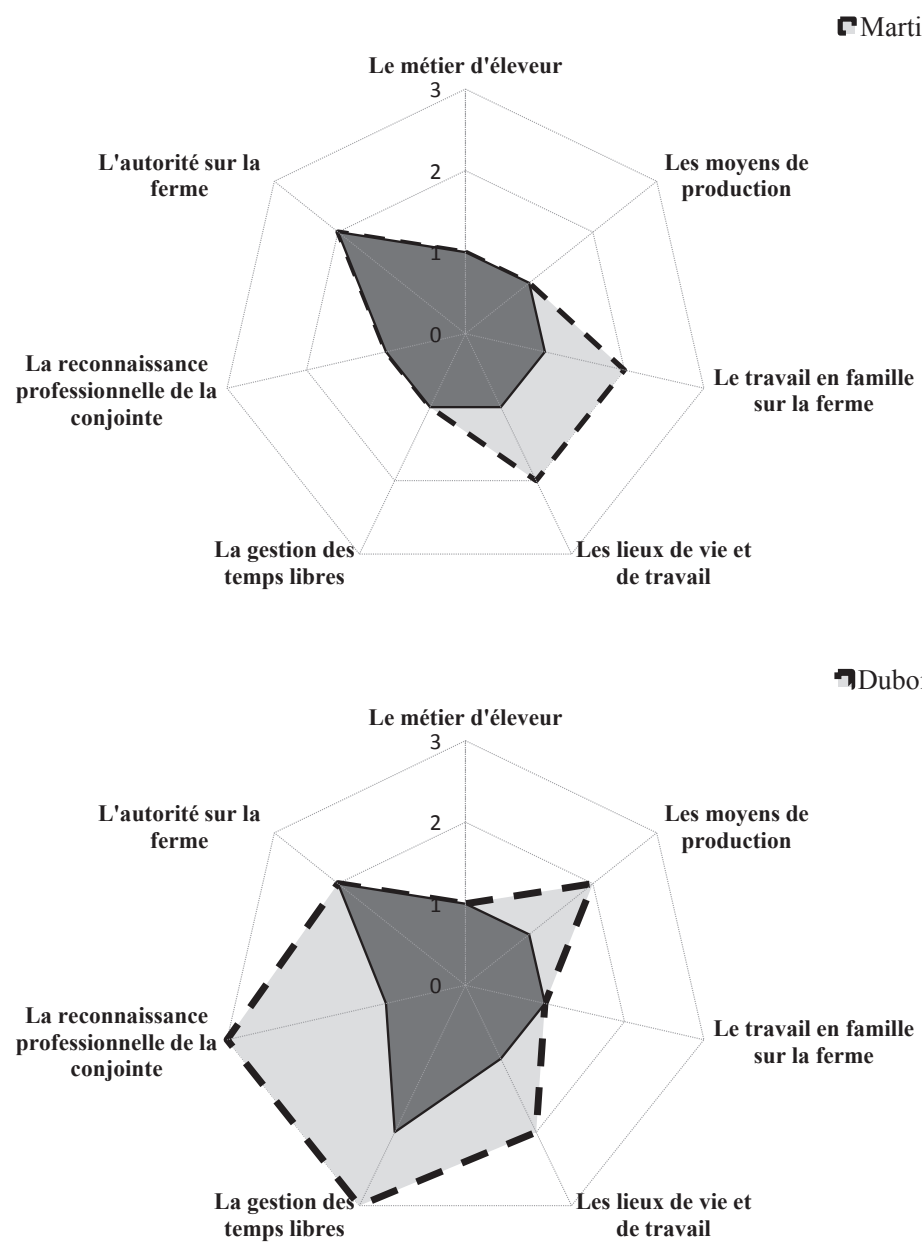

Fig. 2. Les évolutions des rapports familiaux de production sur les exploitations Martin (en haut) et Dubois (en bas). L'aire gris foncé indique la situation initiale, à l'installation de l'éleveur (1984 pour Martin et 1992 pour Dubois), tandis que le périmètre gras pointillé représente la situation actuelle. Les modalités 1, 2 et 3 sont celles des tableaux 1 et 2 . 
On observe ainsi comment, sur la ferme Martin, la famille et l'exploitation demeurent deux mondes étroitement entremêlés, les seules évolutions relevées en plus de 20 ans de trajectoire étant la moindre participation de la famille au travail agricole et la décohabitation générationnelle, évolutions toutes deux liées aux décès des parents de l'éleveur. Dans ces configurations, le lien au patrimoine familial est structurant, et marque profondément à la fois la trajectoire technique de l'exploitation et l'expérience subjective des individus au sein de la famille. Dans nos cas, ce type d'articulation entre la famille et l'exploitation se traduit par une difficile reconnaissance de la contribution féminine au travail agricole, ainsi que par la centralité qu'occupe l'exploitation dans la vie des membres de la famille, en particulier des épouses. Dans ces situations, le collectif, au nom du maintien du patrimoine, gomme en partie les aspirations individuelles des membres de la famille.

Le caractère familial de l'exploitation Dubois s'exprime et a évolué différemment. Tandis qu'au début de la trajectoire, l'exploitation ne différait que peu de l'idéaltype, plusieurs évolutions, liées notamment à l'arrivée de l'épouse sur l'exploitation, témoignent d'une transformation du lien entre la famille et l'exploitation. Cette dernière occupe une place importante dans la vie des membres de la famille (transmission du métier, des moyens de production et de l'autorité, coproduction sur la ferme), mais la représentation qu'en ont ceux-ci differe fortement de la situation précédente. En effet, par des pratiques de distanciation à la ferme, telles que l'instauration d'un cloisonnement des espaces-temps, qui passe aussi par l'individualisation des professions au sein du couple, la famille aménage l'exploitation et les pratiques agricoles pour que cette dernière corresponde à leurs aspirations individuelles.

\section{Discussion-conclusion : diversité et complexité des rapports familiaux de production sur l'exploitation contemporaine}

Entre permanence et transformation, les liens entre la famille et l'exploitation sont ainsi traversés de forces contradictoires. Notre recherche met en évidence que l'exploitation familiale décrite par la sociologie rurale des années 1960 aux années 1970 , et que nous avons érigé en idéal-type, existe encore aujourd'hui. Sur ces fermes, où la dimension patrimoniale est structurante, l'exploitation occupe une place centrale dans la vie des différents membres de la famille, et notamment des femmes. Contribuant souvent au travail agricole sans être statutairement reconnues, leur situation fait écho à ce dont témoigne certaines recherches récentes sur la place des femmes en agriculture (Filippi et Nicourt, 1987 ; Bessière, 2004 ; Rieu, 2004 ; Barthez, 2005 ; Dufour et al., 2010). Dans le même temps, notre recherche rend compte de tendances déjà décrites dans la littérature et qui contribuent à transformer le rapport qu'entretiennent les familles agricoles avec l'exploitation (Giraudet, 2008 ; Dubuisson-Quellier et Giraud, 2010 ; Giraud et Rémy, 2008). C'est ainsi que l'installation hors cadre familial, le salariat agricole ou encore l'emploi salarié des conjointes à l'extérieur de l'exploitation, en introduisant de nouvelles références 
produites en dehors du rapport familial, semblent aller de pair avec une volonté croissante de la part des agriculteurs et de leur famille de séparer la vie familiale des mondes professionnels de chacun, ou encore d'aménager la conduite de la ferme dans la perspective de bénéficier davantage de temps libre pour soi ou en famille.

Cependant, notre grille de lecture met en évidence à l'échelle des exploitations, la grande diversité de pratiques qui traduisent ces tendances. Par exemple, ce n'est pas parce que l'épouse de l'éleveur travaille à l'extérieur que l'exercice de l'agriculture devient un métier comme un autre. L'articulation entre la famille et l'exploitation se joue à de multiples niveaux : la dimension pluri-générationnelle du métier et de l'outil de production, la possibilité ou non de se détacher de cet héritage familial, la répartition du travail et des responsabilités entre les membres de la famille, la reconnaissance de ce travail, la recherche ou non d'un cloisonnement entre les temps et lieux dédiés à la famille et au travail, etc., sont autant d'éléments à considérer pour comprendre à quoi renvoie le caractère familial des exploitations. Entre cloisonnement et imbrication, famille et exploitation s'articulent ainsi selon des modalités diverses nous invitant à penser l'agriculture autrement que de façon dichotomique (entre un modèle familial et un qui ne le serait pas).

Alors qu'il n'existe aujourd'hui plus beaucoup de recherches sur l'exploitation familiale, notre grille de lecture, en mettant en évidence la polysémie du terme " exploitation familiale " dans les Alpes du Nord, est une première étape vers une meilleure caractérisation des dimensions familiales des exploitations. Ce travail exploratoire mériterait d'être mis à l'épreuve sur d'autres terrains et d'autres orientations productives.

\section{Bibliographie}

Agreste, 2012. Recensement agricole 2010 - Main-d'oeuvre et travail agricoles - Un million d'actifs permanents, Agreste Primeur, 276.

Alphandéry, P., Billaud, J.-P., 2009. Retour sur la sociologie rurale. Introduction, Etudes Rurales, 192, 9-22.

Barthez, A., 1982. Famille, travail et agriculture, Paris, Economica.

Barthez, A.,1986. Du labeur paysan au métier d'agriculteur : l'élaboration statistique en agriculture, Cahiers d'économie et sociologie rurales, 3, 45-72.

Barthez, A., 1999. Installation " hors du cadre familial " et relation d'adoption, Économie Rurale, 253, 15-20.

Barthez, A., 2005. Devenir agricultrice : à la frontière de la vie domestique et de la profession, Économie rurale, 289-290, 30-43.

Bertaux, D., 1977. Destins personnels et structures de classes, Paris, PUF.

Bessière, C., 2003. Une profession familiale : les trois dimensions de la vocation agricole., in G. Séverine, A. Gramain, F. Weber (Eds), Charges de famille. Dépendance et parenté dans la France contemporaine, Paris, La Découverte, 237-272. 
Bessière, C., 2004. «vaut mieux qu'elle travaille à l'extérieur ! " Enjeux du travail salarié des femmes d'agriculteurs dans les exploitations familiales, Les cahiers du genre, 37, 93-114.

Bessière, C., 2006. Maintenir une entreprise familiale. Enquête sur les exploitations viticoles de la région délimitée Cognac, Thèse de doctorat en Sociologie, Université Paris V. Bessière, C., 2008. "Travailler à l'extérieur " : des implications ambivalentes pour les compagnes d'agriculteurs, Nouvelles Questions Féministes, 27, 2, Lambivalence du travail : entre exploitation et émancipation (2008), pp. 53-66.

Bessière, C., 2010. De génération en génération. Arrangements de famille dans les entreprises viticoles de Cognac, Paris, Raisons d'agir.

Bessière, C., Giraud, C., Renahy, N., 2008. Famille, travail, école et agriculture, Revue d'Études en agriculture et environnement, 88, 5-19.

Bjorkhaug, H., Blekesaune, A., 2008. Gender and work in Norwegian family farm business, Sociologia ruralis, 48, 2, 152-165.

Bosse-Platière, H., 2005. L'avenir familial de l'exploitation agricole, Économie rurale, 289-290, 10-29.

Cardon, P., 2004. Des femmes et des fermes. Genres, parcours biographiques et transmission familiale, Paris, L'Harmattan.

Dahache, S., 2012. La féminisation de l'enseignement agricole, Sociologie des rapports de genre dans le champ des formations professionnelles, Paris, L'Harmattan.

De Singly, F., 2005. Le soi, le couple et la famille, Paris, Armand colin.

Dedieu, B., Chabosseau, J.-M., Willaert, J., Benoît, M., Laignel, G., 1998. L'organisation du travail dans les exploitations d'élevage : une méthode de caractérisation en élevage ovin du centre ouest, Études et recherches sur les systèmes agraires et le développement, 31, 63-80.

Delphy, C., 1983. Agriculture et travail domestique : la réponse de la bergère à Engels, Nouvelles questions féministes, 5, 3-18.

Dubuisson-Quellier, S., Giraud, C., 2010. Les agriculteurs entre clôtures et passerelles, in Hervieu, B., Mayer, N., Muller, P., Purseigle, F., Rémy, J. (Eds), Les mondes agricoles en politique, Paris, Presses de SciencesPo, 111-129.

Dufour, A., Courdin, V., Dedieu, B., 2010. Femmes et travail en couple : pratiques et représentations en élevage laitier en Uruguay et en France, Cahiers agricultures, 19, 5, 371-376.

Filippi, G., Nicourt, C., 1987. Domestique-professionnel : la cohérence du travail des femmes dans les exploitations agricoles familiales, Économie rurale, 178179, 47-52.

Gasson, R., Crow, G., Errington, A., Hutson, J., Mardsen, T., Winter, D.-M., 1993. The farm as a family business : a review, Journal of Agricultural Economics, 39, $1,1-41$.

Giraud, C. , 2006. La famille devient individualiste, Constructif, 14.

Giraud, C., Rémy, J., 2008. Les choix des conjoints en agriculture, Revue d'Étude en Agriculture et Environnement, 88, 3, 14-46. 
Gramain, A., Weber, F., 2003. Introduction. Modéliser l'économie domestique, in Gojard, S., Gramain, A., Weber, F. (Eds), Charges de famille, Paris, La découverte, 9-42.

Jacques-Jouvenot, D., 1997. Choix du successeur et transmission patrimoniale, Paris, L'Harmattan.

Jacques-Jouvenot, D., Gillet, M., 2001. L'agriculture en Franche-Comté : un métier patrimonial rediscuté, Études Rurales, 159-160, 111-128.

Madelrieux, S., Dupré, L., Rémy, J., 2009. Itinéraires croisés et relations entre éleveurs et salariés dans les Alpes du Nord, Économie rurale, 313-314, 6-23.

Mundler, P., Rémy, J., 2012. L'exploitation familiale à la française : une institution dépassée ? L'Homme et la société, 183-184, 161-179.

Olivier-Salvagnac, V., Legagneux, B., 2012. Lagriculture de firme : un fait émergeant dans le contexte agricole français ? Études rurales, 190, 77-97.

Ollagnon, H., 1989. Une approche patrimoniale de la qualité du milieu naturel, in Mathieu, N., Jollivet, M. (Eds), Du rural à l'environnement : la question de la nature aujourd'hui, Paris, L'Harmattan, 259-268.

Petit, M., 2006. L'exploitation agricole familiale : leçons actuelles de débats anciens, Cahiers agricultures, 15, 6, 486-490.

Purseigle, F., 2012. Introduction, Études rurales, 190, 19-23.

Rémy, J., 1987. La crise de professionalisation en agriculture : les enjeux de lutte pour le contrôle du titre d'agriculteur, Sociologie du travail, 29, 4, 415-441.

Rémy, J., 2010. Un métier en transformation. Introduction, in Perrineau, P., Mossuz-Lavau, J., (Eds), Les mondes agricoles en politique, Paris, Presses de SciencesPo, 41-50.

Rieu, A.-M., 2004. Agriculture et rapports sociaux de sexe. La " révolution silencieuse " des femmes en agriculture, Cahiers du genre, 2, 37, 115-130.

Salifios-Rothschilf, C.,1976. A macro and micro-examination of family power and love : an exchange model, Journal of Marriage and the Family, 38, 2, 355-362.

Salmona, M., 1994. Les paysans français. Le travail, les métiers, la transmission des savoirs, Paris, L'harmattan.

Terrier, M., 2013. Les réalités de l'exploitation agricole familiale au prisme du temps long. Proposition d'un cadre d'analyse interdisciplinaire et illustrations en exploitations d'elevage bovin lait dans le Vercors, Thèse de doctorat en zootechnie système et en sociologie, Inra-Sad, Irstea, Paris, AgroParisTech.

Terrier, M., Madelrieux, S., Dufour, A., Dedieu, B., 2012. Saisir la diversité des formes d'articulation entre la famille et l'exploitation : une grille de lecture, Revue d'études en agriculture et environnement, 93, 3, 299-322.

Van de Walle, I., 1993. Terre des hommes, salaire des femmes. Stratégies individuelles et familiales en agriculture. Le cas du Calvados, Thèse de doctorat de sociologie, Paris, École des hautes études en sciences sociales. 